\title{
A DYNAMICALLY ADAPTIVE LATTICE BOLTZMANN METHOD FOR THERMAL CONVECTION PROBLEMS
}

\author{
KAI FELDHUSEN ${ }^{a, b}$, RALF DEITERDING $^{c, *}$, Claus WAGNER $^{a, b}$ \\ ${ }^{a}$ Institute of Aerodynamics and Flow Technology \\ German Aerospace Center (DLR), Bunsenstrasse 10, 37073 Göttingen, Germany \\ ${ }^{b}$ Institute of Thermodynamics and Fluid Mechanics \\ University of Technology in Ilmenau, Helmholtzring 1, 98693 Ilmenau, Germany \\ ${ }^{c}$ Aerodynamics and Flight Mechanics Research Group \\ University of Southampton, Highfield Campus, Southampton SO17 1BJ, UK \\ e-mail: r.deiterding@soton.ac.uk
}

\begin{abstract}
Utilizing the Boussinesq approximation, a double-population incompressible thermal lattice Boltzmann method (LBM) for forced and natural convection in two and three space dimensions is developed and validated. A block-structured dynamic adaptive mesh refinement (AMR) procedure tailored for the LBM is applied to enable computationally efficient simulations of moderate to high Rayleigh number flows which are characterized by a large scale disparity in boundary layers and free stream flow. As test cases, the analytically accessible problem of a two-dimensional (2D) forced convection flow through two porous plates and the non-Cartesian configuration of a heated rotating cylinder are considered. The objective of the latter is to advance the boundary conditions for an accurate treatment of curved boundaries and to demonstrate the effect on the solution. The effectiveness of the overall approach is demonstrated for the natural convection benchmark of a 2D cavity with differentially heated walls at Rayleigh numbers from $10^{3}$ up to $10^{8}$. To demonstrate the benefit of the employed AMR procedure for three-dimensional (3D) problems, results from the natural convection in a cubic cavity at Rayleigh numbers from $10^{3}$ up to $10^{5}$ are compared with benchmark results.
\end{abstract}

Keywords: lattice Boltzmann method, adaptive mesh refinement, thermal convection, incompressible.

\section{Introduction}

In recent years, the lattice Boltzmann method (LBM) has emerged as a powerful alternative to traditional Navier-Stokes (NS) solvers (Chen and Doolen, 1998) to predict thermal fluid flow (Guo et al., 2002; Kuznik et al., 2007; Peng et al., 2003), turbulent fluid flow (Jonas et al., 2006), multiphase fluid flow (Lee and Lin, 2005; Yu and Fan, 2009) and magnetohydrodynamics (Deller, 2002). Instead of discretizing the NS equations directly, the LBM is based on solving a simplified version of the Boltzmann equation in a specifically chosen discrete phase space. Using a Chapman-Enskog expansion, it has been shown that the approach recovers the NS equations in the limit of a vanishing Knudsen number (Hähnel, 2004). Originally proposed for the isothermal weakly

\footnotetext{
*Corresponding author
}

compressible case, several method enhancements for incompressibility (He and Luo, 1997; Qian et al., 1992) as well as incorporation of a buoyancy-driven temperature field for thermal convection flows are available (He et al., 1998; Qian, 1993). In general, there are two different categories of thermal lattice Boltzmann models. For the multispeed approach, the number of discrete velocity directions will be increased and the equilibrium distribution function is supplemented by higher order velocity terms to solve the internal energy equation (cf. McNamara and Alder, 1993; Alexander et al., 1993; Qian, 1993). However, this model is reported to exhibit numerical instabilities (cf. Chen and Teixeira, 2000). Here, we have chosen to pursue the strictly incompressible double distribution function (DDF) approach proposed by Guo et al. (2002) for 2D and the straightforward expansion to 3D by He et al. (2004) as well as Azwadi 
Che Sidik and Syahrullail (2009).

While the original LBM is formulated on a uniform Cartesian grid, an increase in the local resolution is particularly necessary in the thermal boundary layers close to heated objects and walls. Kuznik et al. (2007) and Peng et al. (2003) demonstrated the computational benefit of a non-uniform grid for a thermal DDF LBM in two and three spatial dimensions for simulating thermal convection in Cartesian cavities. In both works, a static geometry transformation is applied to the discretization in order to stretch the Cartesian lattice in the cavity center and reduce the spacing continuously towards the walls. Solution adaptive meshing is not used and on-the-fly mesh adaptation seems to have been applied so far to DDF LBMs only in the context of isothermal two-phase flows (cf. Yu and Fan, 2009). Our objective in this paper is to close this gap. We supplement a thermal DDF LBM method with a solution adaptive, dynamic mesh refinement. While adaptive lattice Boltzmann methods in the past have used primarily isotropic refinement of individual cells) (cf., e.g., Chen et al., 2006), we apply in here a block-based approach, which is more suitable for the regular transport step of the LBM and thereby computationally significantly more efficient. The underlying data structures including distributed memory parallelization are borrowed from the finite volume mesh refinement system AMROC (Deiterding, 2011).

In order to fit smoothly into AMROC, the DDF LBM is formulated on cell-centered data structures and not node-based, as it is mainly used for the LBM in order to simplify the implementation of physical boundary conditions. In addition, a complex geometry boundary condition treatment for possibly moving structures is incorporated. The update of the non-uniform lattice and the dynamic refinement procedure are orchestrated with the recursive Berger-Collela algorithm (Berger and Colella, 1988). While the efficiency of this algorithm is undisputed for time-explicit finite volume schemes, its application to the LBM is a novelty. In summary, our adaptive method is uniquely designed for efficient simulation of real-world thermal flow problems. In this paper, the underlying computational techniques are described and the required validation for well-understood thermal convection problems is provided.

In Section 2, we discuss the details of the numerical method, including the advanced thermal lattice Boltzmann approach, the block-based AMR method and the treatment of geometrically complex boundaries in the originally Cartesian scheme. Section 3 presents the computational results, where the analytic solution of the 2D flow between two moving porous plates, the 2D flow around a rotating heated cylinder and the well-known benchmark case of a two-dimensional cavity with differentially heated walls are considered. The result section is closed presenting the solution of the flow in a $3 \mathrm{D}$ cubic cavity with differentially heated walls. The conclusions, including a short outlook, are given in Section 4 .

\section{Numerical method}

2.1. Thermal lattice Boltzmann scheme. The incompressible two-dimensional LBM constructed under the Boussinesq approximation used in the present work has been proposed by Guo et al. (2002). For the three-dimensional case, the incompressible LBM operator by $\mathrm{He}$ et al. (2004) is applied. By using the Bhatnagar-Gross-Krook (BGK) collision model (Bhatnagar et al., 1954), the lattice Boltzmann equation for the partial probability distribution function $f_{i}$ with force field term $F_{i}$ can be formulated as

$$
\begin{aligned}
f_{i}\left(\mathbf{x}+c \mathbf{e}_{i} \Delta t, t+\Delta t\right) & =f_{i}(\mathbf{x}, t) \\
& -\frac{1}{\tau_{\nu}}\left(f_{i}(\mathbf{x}, t)-f_{i}^{(\mathrm{eq})}(\mathbf{x}, t)\right)+\Delta t F_{i} .
\end{aligned}
$$

In the DDF approach, a set of the corresponding lattice Boltzmann equations

$$
\begin{aligned}
g_{i}(\mathbf{x}+ & \left.c \mathbf{e}_{i} \Delta t, t+\Delta t\right) \\
& =g_{i}(\mathbf{x}, t)-\frac{1}{\tau_{\mathcal{D}}}\left(g_{i}(\mathbf{x}, t)-g_{i}^{(\mathrm{eq})}(\mathbf{x}, t)\right)
\end{aligned}
$$

is introduced based on distribution functions $g_{i}$ that are used to convect the macroscopic scalar quantity, here temperature, with the flow field. In the latter, $\mathbf{e}_{i}$ is the unit velocity vector in the direction of the $i$-th discrete velocity space direction, $t$ and $\Delta t$ denote the time and time step, $x$ the position, $\Delta x$ the spatial increment, and $c=\Delta x / \Delta t$ is the particle speed. The relaxations times are $\tau_{\nu}$ for the flow field and $\tau_{\mathcal{D}}$ for the temperature field. The respective equilibrium distribution functions are denoted by $f_{i}^{\text {(eq) }}$ and $g_{i}^{\text {(eq) }}$. In the two-dimensional case, a model with nine discrete unit velocities is used to compute the flow field (D2Q9) and an operator with four discrete velocities for the temperature field (D2Q4). The orientation of the discrete unit length velocities $\mathbf{e}_{i}$ used to compute the velocity fields is depicted in Fig. 1

In the three-dimensional case, an operator with nineteen unit velocities is used for the flow field (D3Q19) and a model with six discrete velocities for the temperature field (D3Q6). The extended version of the orientation of the discrete unit length velocities $\mathbf{e}_{i}$

$$
\mathbf{e}_{i}= \begin{cases}(0,0), & i=0, \\ ( \pm 1,0,0),(0, \pm 1,0),(0,0, \pm 1), & i=1, \ldots, 6, \\ ( \pm 1, \pm 1,0),( \pm 1,0, \pm 1),(0, \pm 1, \pm 1), & i=7, \ldots, 18,\end{cases}
$$

The basic LBM algorithm is divided into the steps of transport (or streaming) and collision, which are applied basically identically to (1) and (2). The following 


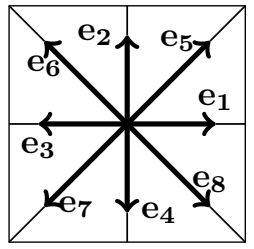

Fig. 1. Numerical stencil of D2Q9: discrete velocity directions in a computational cell.

transport step represents the advection of fluid particles along the corresponding discrete velocities:

$$
\mathcal{T}: \quad \tilde{f}_{i}\left(\mathbf{x}+c \mathbf{e}_{i} \Delta t, t+\Delta t\right)=f_{i}(\mathbf{x}, t) .
$$

Relaxation of the distribution functions towards the local equilibrium is performed on the transported distribution functions in the collision step

$$
\begin{aligned}
\mathcal{C}: \quad f_{i}(\cdot, t+\Delta t) & =\tilde{f}_{i}(\cdot, t+\Delta t) \\
& -\frac{1}{\tau_{\nu}}\left(\tilde{f}_{i}(\cdot, t)-\tilde{f}_{i}^{(\mathrm{eq})}(\cdot, t)\right) .
\end{aligned}
$$

With the pressure $p$ and the velocity vector $\mathbf{u}$ as independent variables, the specific equilibrium distribution function $f_{i}^{(\mathrm{eq})}$ for the D2Q9 model is defined as (Guo et al., 2002)

$$
f_{i}^{(\mathrm{eq})}= \begin{cases}-4 \sigma \frac{p}{c^{2}}-s_{i}(\mathbf{u}) & \text { for } i=0 \\ \lambda \frac{p}{c^{2}}+s_{i}(\mathbf{u}) & \text { for } i=1, \ldots, 4 \\ \gamma \frac{p}{c^{2}}+s_{i}(\mathbf{u}) & \text { for } i=5, \ldots, 8\end{cases}
$$

where the parameters $\sigma, \lambda$, and $\gamma$ satisfy $\lambda+\gamma=\sigma$ and $\lambda+2 \gamma=1 / 2$. The functions $s_{i}(\mathbf{u})$ depend on the macroscopic velocity vector $\mathbf{u}$ and the discrete velocity vector $\mathbf{e}_{i}$ and obey

$$
s_{i}(\mathbf{u})=\omega_{i}\left[3 \frac{\mathbf{e}_{i} \cdot \mathbf{u}}{c}+4.5 \frac{\left(\mathbf{e}_{i} \cdot \mathbf{u}\right)^{2}}{c^{2}}-1.5 \frac{|\mathbf{u}|^{2}}{c^{2}}\right],
$$

where the coefficients are $\omega_{0}=4 / 9, \omega_{1, \ldots, 4}=1 / 9$, and $\omega_{5, \ldots, 8}=1 / 36$. Using (6) and (7), the macroscopic values for velocity and dynamic pressure are given as

$$
\mathbf{u}=\sum_{i>0} c \mathbf{e}_{i} f_{i}, \quad p=\frac{c^{2}}{4 \sigma}\left[\sum_{i>0} f_{i}+s_{0}(\mathbf{u})\right] .
$$

For the D3Q19 model, the parameters change to $\sigma=$ $1 / 2, \lambda=1 / 18$, and $\gamma=1 / 36$. Furthermore, the weight coefficients are given by $\omega_{0}=1 / 3, \omega_{1, \ldots, 6}=1 / 18$, and $\omega_{7, \ldots, 18}=1 / 36$. For the D2Q4 model used to compute the temperature field, the equilibrium function $g_{i}^{(\mathrm{eq})}$ is

$$
g_{i}^{(\mathrm{eq})}=\frac{T}{4}\left[1+2 \frac{\mathbf{e}_{i} \cdot \mathbf{u}}{c}\right], \quad i=1, \ldots, 4,
$$

and the macroscopic temperature is $T=\sum_{i=1}^{4} g_{i}$.

Analogously, in the D3Q6 model of the temperature field, the equilibrium function reads

$$
g_{i}^{(\mathrm{eq})}=\frac{T}{6}\left[1+3 \frac{\mathbf{e}_{i} \cdot \mathbf{u}}{c}\right], \quad i=1, \ldots, 6,
$$

and the macroscopic temperature $T=\sum_{i=1}^{6} g_{i}$.

Since the fluid is assumed to be incompressible, a linear dependency between temperature differences and gravitational forces is applied (Boussinesq approximation) (cf. Mohamad and Kuzmin, 2010), which leads to the force term $F_{i}$. The force in 111 acts only in the two direct vertical directions. For 2D, this can be expressed according to Fig.1(Guo et al., 2002) as

$$
F_{i}=\frac{1}{2}\left(\delta_{i 2}+\delta_{i 4}\right) \mathbf{e}_{i} \cdot \mathbf{F}
$$

with

$$
\mathbf{F}=\mathbf{g} \beta\left(T-T_{\mathrm{ref}}\right),
$$

where $\mathbf{g}$ and $\beta$ are the acceleration vector of gravity and the coefficient of thermal expansion, respectively, and $T_{\text {ref }}$ is the average temperature. The force term establishes the coupling between the lattice Boltzmann equations for the flow field (1) and the temperature field (2).

Note that through a multiscale Chapman-Enskoq expansion, the incompressible Navier-Stokes equations can be derived from the discussed incompressible LBGK model. After neglecting the viscous heat dissipation and compression work carried out by the pressure, the temperature field obeys a passive scalar equation. Accordingly, the approximated incompressible equations in this work are (cf. Guo et al., 2002),

$$
\begin{aligned}
\nabla \cdot \mathbf{u} & =0 \\
\frac{\partial \mathbf{u}}{\partial t}+\nabla \cdot(\mathbf{u u}) & =-\nabla p+\nu \nabla^{2} \mathbf{u}+\mathbf{F} \\
\frac{\partial T}{\partial t}+\nabla \cdot(\mathbf{u} T) & =\mathcal{D} \nabla^{2} T
\end{aligned}
$$

The kinematic viscosity $\nu$ and the thermal diffusivity $\mathcal{D}$ are related to the dimensionless collision times by $\nu=$ $\frac{1}{6}\left(2 \tau_{\nu}-1\right) c \Delta x$ and $\mathcal{D}=\frac{1}{4}\left(2 \tau_{\mathcal{D}}-1\right) c \Delta x$. Introducing the physical speed of sound as $c_{s}=c / \sqrt{3}$, these expressions yield the relations

$$
\tau_{\nu}=\frac{\nu+c_{s}^{2} \Delta t / 2}{c_{s}^{2} \Delta t}, \quad \tau_{\mathcal{D}}=\frac{\mathcal{D}+\frac{3}{2} c_{s}^{2} \Delta t / 2}{\frac{3}{2} c_{s}^{2} \Delta t},
$$

which can be used to evaluate the dimensionless collision times in (11) and (2) for given macroscopic gas properties $\nu, \mathcal{D}$ and time step $\Delta t$.

2.2. Adaptive mesh refinement. For local dynamic mesh adaptation, we have adopted the block-structured 
AMR method proposed by Berger and Colella (1988). This method was originally designed for time-explicit finite volume schemes for hyperbolic conservation laws; however, its recursive execution procedure and natural consideration of time step refinement make it equally applicable to lattice Boltzmann schemes, which is not surprising as a hyperbolic constant velocity advection equation is the theoretical underpinning of the transport step (4). In order to fit smoothly into our existing, fully parallelized finite volume AMR software system AMROC (Deiterding, 2011), we have implemented the cell-based LBM. In the block-based AMR approach, finite volume cells are clustered with a special algorithm into non-overlapping rectangular grids. The grids have a suitable layer of halo cells for synchronization and applying inter-level and physical boundary conditions. Refinement levels are integrated recursively starting from the coarsest level. With index $l$ denoting the AMR level, the spatial mesh width $\Delta x_{l}$ and the time step $\Delta t_{l}$ are refined by the same factor $r_{l}$, where we assume $r_{l} \geq 2$ for $l>0$ and $r_{0}=1$.

In the adaptive thermal LBM, it is of foremost importance that the dimensionless collision times of the DDF LBM be adjusted on a level basis according to (16) as the time step is recursively refined. In addition, the interface region requires a specialized treatment to ensure consistent transport of coarse-grid distributions into refined cells and of fine-grid distributions into the coarse cells adjacent to the boundaries of refined regions. Since the D2Q4 stencil is just a simplified version of the D2Q9 method, we restrict our description of the interface algorithm to the latter. Distinguishing between the transport and collision operators, respectively, $\mathcal{T}$ and $\mathcal{C}$ (cf. (4) and (5)), our method proceeds in the following steps if a refinement factor of 2 is considered:

1. Complete the update on the coarse grid: $f_{i}^{C, n+1}:=$ $\mathcal{C} \mathcal{T}\left(f_{i}^{C, n}\right)$.

2. Use coarse grid distributions $f_{i \text {,in }}^{C, n}$ that propagate into the fine grid (cf. Fig. 2(a)), to construct an initial fine grid halo values $f_{i, \text { in }}^{F, n}$ (cf. Fig. 2(b)).

3. Complete transport $\tilde{f}_{i}^{F, n}:=\mathcal{T}\left(f_{i}^{F, n}\right)$ on the whole fine mesh. Collision $f_{i}^{F, n+1 / 2}:=\mathcal{C}\left(\tilde{f}_{i}^{F, n}\right)$ is applied only in the interior cells (grey in Fig. 2(b)).

4. Repeat Step 3 to obtain $\tilde{f}_{i}^{F, n+1 / 2}:=\mathcal{T}\left(f_{i}^{F, n+1 / 2}\right)$ and $f_{i}^{F, n+1}:=\mathcal{C}\left(\tilde{f}_{i}^{F, n+1 / 2}\right)$.

5. Average outgoing distributions from fine grid halos (Fig. 2(c)), that is, $\tilde{f}_{i, \text { out }}^{F, n+1 / 2}$ in the inner halo layer and $\tilde{f}_{i \text {,out }}^{F, n}$ (outer halo layer), to obtain $\tilde{f}_{i, \text { out }}^{C, n}$.
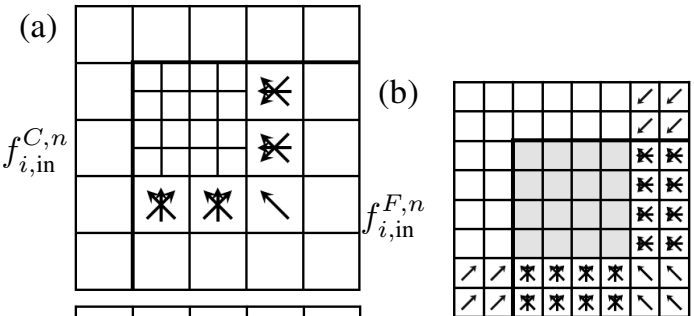

(c)
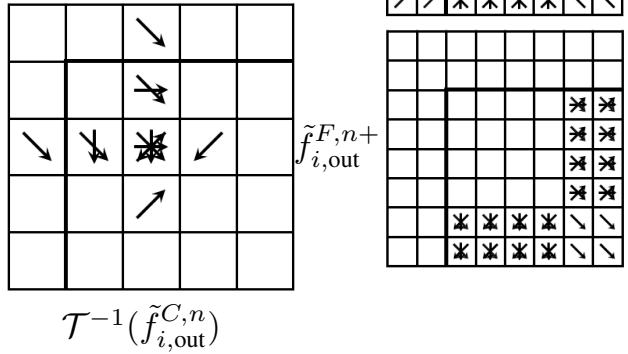

Fig. 2. Visualization of distributions involved in data exchange at coarse $(C)$ and fine $(F)$ boundaries. The thick black lines indicate a physical boundary. Coarse distributions going into fine grid (a), incoming interpolated fine distributions in halos (top) and outgoing distributions in halos after two fine-level transport steps (bottom) (b), averaged distributions replacing coarse values before update is repeated in cells next to boundary (c).

6. Revert transport for averaged outgoing distributions, $\bar{f}_{i \text {,out }}^{C, n}:=\mathcal{T}^{-1}\left(\tilde{f}_{i, \text { out }}^{C, n}\right)$, and overwrite those in the previous coarse grid time step.

7. Synchronization of $f_{i}^{C, n}, \bar{f}_{i, \text { out }}^{C, n}$ on the entire level.

8. Repeat complete update on coarse grid cells next to the coarse-fine boundary only: $f_{i}^{C, n+1}:=$ $\mathcal{C} \mathcal{T}\left(f_{i}^{C, n}, \bar{f}_{i, \text { out }}^{C, n}\right)$.

In this description and in Fig. 2, the time steps on the coarse level $C$ are indexed by the superscript $n$, index $F$ denotes the fine level, and the subscripts in and out indicate distributions which are convected in- and outwards of the fine grid along the coarse-fine boundary. The overall algorithm is computationally equivalent to the method by Chen et al. (2006) but explicitly tailored to the Berger-Collela recursion that updates coarse grids in their entirety before fine grids are computed. The complete update of the entire respective coarse mesh and subsequent correction is the basis of the computational efficiency of the Berger-Collela method; however, this approach has so far hardly been applied to lattice Boltzmann methods. Previous adaptive LBMs, (cf. Chen et al., 2006), update the fine grid before the respective coarse level and provide no apparent avenue for implementing time-interpolated fine level interface conditions. While not being used above, the benefit of interpolating in time the non-equilibrium portion of coarse-grid distributions crossing the coarse-fine interface in Step 4 has been demonstrated by Dupuis and Chopard 
(2003) and will be considered in our implementation in the future.

2.3. Wall boundary treatment. Correct implementation of the boundary condition is very important for numerical stability. For the test cases considered, we need different implementations of boundary conditions for the velocity and temperature partial distribution functions. No-slip or adiabatic boundary conditions are realized via a bounce-back approach for the unknown partial distribution functions as described by Succi (2001). To prescribe fixed macroscopic values on the wall in the form of Dirichlet boundary conditions, we use a second order extrapolation scheme by Guo et al. (2002). The outflow boundary conditions are implemented via a linear propagation as prescribed by Mohamad (2011). We use a set of halo cells around the computational domain to manipulate the unknown partial probability distribution functions in the transport step.

2.4. Curved boundary treatment. We represent non-Cartesian boundaries implicitly on the adaptive Cartesian grid by utilizing a scalar level set function $\varphi$ that stores the distance to the boundary surface. The boundary surface is located exactly at $\varphi=0$, and the boundary outer normal at each mesh point can be evaluated as $\mathbf{n}=-\nabla \varphi /|\nabla \varphi|$ (cf. Deiterding, 2011). We treat a fluid cell as an embedded ghost cell if its midpoint satisfies $\varphi<0$. In order to implement non-Cartesian boundary conditions with the LBM, we have chosen to pursue for now a first order accurate ghost fluid approach. In our technique, the density distributions in embedded ghost cells are adjusted to model the boundary conditions of a non-Cartesian reflective wall moving with velocity vector $\mathbf{w}$ before applying the unaltered LBM. The last step involves interpolation and mirroring of $p, T, \mathbf{u}$, across the boundary to $p^{\prime}, T^{\prime}$ and $\overline{\mathbf{u}}$, and modification of the macroscopic velocity vector in the immersed boundary cells to $\mathbf{u}^{\prime}=2 \mathbf{w}-\overline{\mathbf{u}}$ (cf. Deiterding, 2011). From the newly constructed macroscopic values the distributions in the embedded ghost cells are simply set to $f_{i}^{\text {eq }}\left(p^{\prime}, \mathbf{u}^{\prime}\right)$ and $g_{i}^{\mathrm{eq}}\left(T^{\prime}\right)$.

\section{Results}

For the setup of physical configurations, it is useful to recall the definitions of the dimensionless Rayleigh and Prandtl number, which are

$$
\mathrm{Ra}=\frac{g \beta \Delta T H^{3}}{\nu \mathcal{D}}, \quad \operatorname{Pr}=\frac{\nu}{\mathcal{D}} .
$$

The characteristic velocity $U$ for thermal convection flows is generally set to the buoyancy velocity $U=\sqrt{g \beta \Delta T H}$, where $H$ denotes a problem-dependent geometric height.
A cell $(j, k)$ is flagged for refinement if any of the scaled gradient relations

$$
\begin{gathered}
\left|\phi_{j+1, k}-\phi_{j, k}\right|>\epsilon_{\phi}, \quad\left|\phi_{j, k+1}-\phi_{j, k}\right|>\epsilon_{\phi}, \\
\left|\phi_{j+1, k+1}-\phi_{j, k}\right|>\epsilon_{\phi}
\end{gathered}
$$

is satisfied for a particular macroscopic component $\phi_{j, k}$ and a prescribed limit $\epsilon_{\phi}$. If not stated otherwise, $\epsilon_{T}$ is set to $1 \%$ of the maximum temperature and $\epsilon_{u}, \epsilon_{v}, \epsilon_{w}$ are set to $5 \%$ of the characteristic velocity.

3.1. Porous plate. In order to validate the basic numerical method, we selected the problem of forced thermal convection between two porous plates also employed by Guo et al. (2002). This problem is set up as a Couette flow between two porous plates of which the upper is in motion. A constant flow is injected normal to the lower plate and leaves the domain through the top plate with the same rate. The bottom plate is cooled, while the upper plate is heated. The analytic solutions for the horizontal velocity and the temperature profile in steady state are

$$
\begin{aligned}
& u^{*}(y)=U_{0}\left(\frac{e^{\operatorname{Re} \cdot y / H}-1}{e^{\operatorname{Re}}-1}\right), \\
& T^{*}(y)=T_{C}+\Delta T\left(\frac{e^{\operatorname{RePr} \cdot y / H}-1}{e^{\operatorname{RePr}}-1}\right),
\end{aligned}
$$

where $U_{0}$ is the velocity of the upper plate. The Reynolds number Re is based on the injection velocity $V_{0}$ and is given by $\operatorname{Re}=V_{0} \cdot H / \nu$. We study three different configurations with a varying Reynolds number. The Prandtl number is fixed and set to $\operatorname{Pr}=0.71$, which corresponds to air, and the Rayleigh number is set to $\mathrm{Ra}=100$. The velocity of the upper plate is also fixed and set to $U_{0}=0.1$. Finally, the dimensionless relaxation time $\tau_{\nu}$ on the coarsest level is prescribed as $\tau_{\nu}=1 / 1.25$.

The simulations are performed for the Reynolds numbers $\operatorname{Re}=5,10$ and 20 using a base grid of $64 \times 32$ cells. The successive embedded static refinement with four additional levels with refinement factors $r_{1, \ldots, 4}=4$ is realized in the complete computational domain $[0,64] \times$ $[0,32]$. In detail, we have the finest resolution $r_{4}$ near the top and bottom boundaries $[0,64] \times([0,4] \cup[28,32])$, then $r_{2}$ in $[0,64] \times([4,8] \cup[24,28])$ and $r_{3}$ in $[0,64] \times$ $([8,12] \cup[20,24])$. The coarsest refinement level $r_{1}$ is in the center region $[0,64] \times[12,20]$. The entire velocity field is initialized at rest as $(0,0)^{T}$ and the temperature field to the constant value $T_{C}$.

We compare the numerical predictions of the velocity and temperature distributions with the analytic solution. Figure 3 plots the normalized numerical results vs. the analytic solutions. From the point of view of validation, the macroscopic values for the horizontal velocity and scalar temperature are being calculated in each cell 

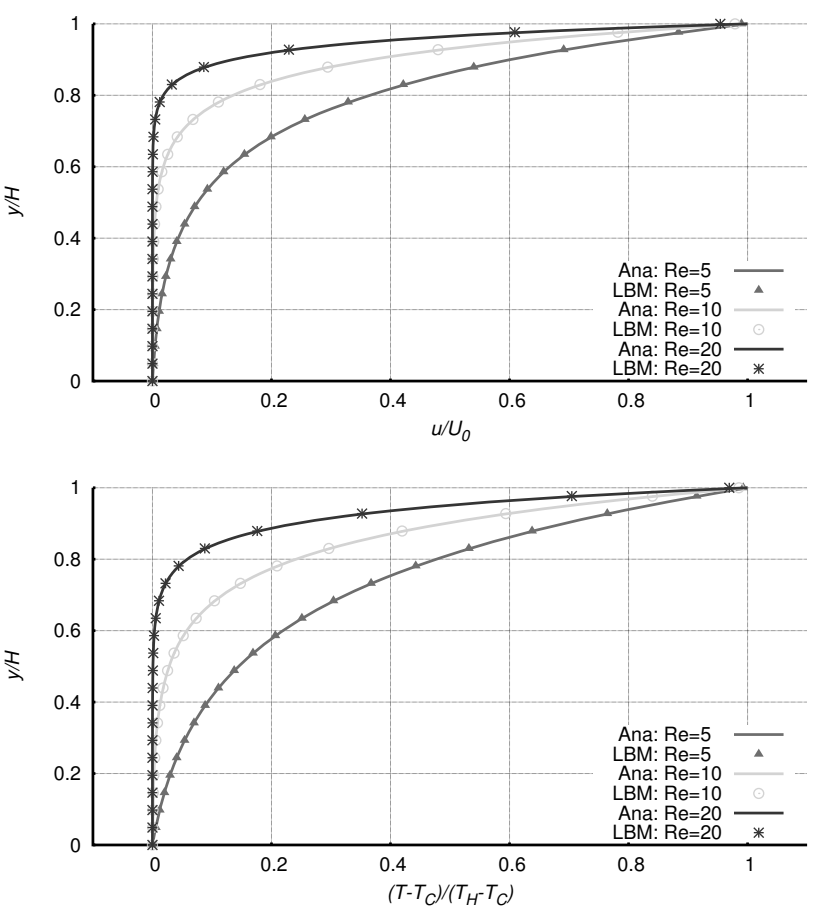

Fig. 3. Comparison of velocity and temperature distribution predicted for different values of Re in comparison with the analytic solution.
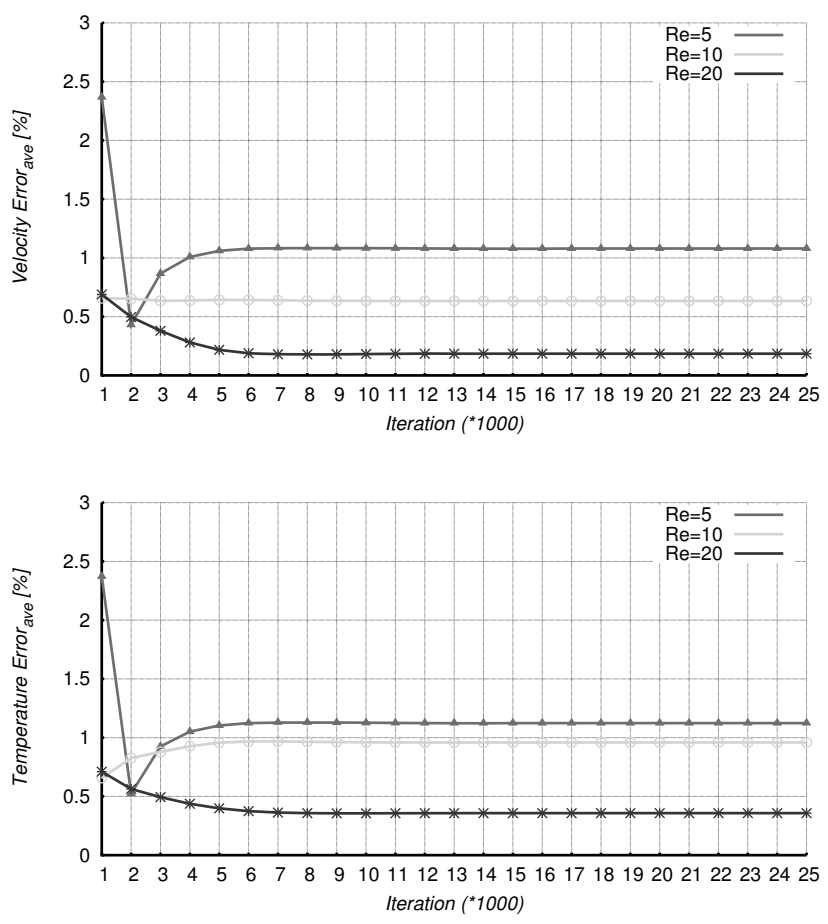

Fig. 4. Averaged L2-norm error for computed macroscopic velocity and temperature over iteration steps for different values of Re.

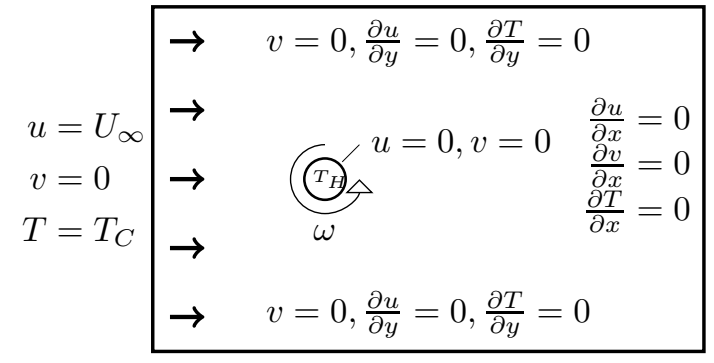

Fig. 5. Setup for the flow past a heated rotating cylinder.

midpoint along each vertical line. The macroscopic values in the cells are averaged along the horizontal lines. The L2-norm errors of the averaged macroscopic quantities $\Phi$ are calculated with

$$
E_{\mathrm{ave}}(\Phi)=\frac{\sqrt{\sum_{i}\left|\Phi_{\mathrm{ave}}\left(\mathbf{x}_{i}\right)-\Phi^{*}\left(\mathbf{x}_{i}\right)\right|^{2}}}{\sqrt{\sum_{i}\left|\Phi^{*}\left(\mathbf{x}_{i}\right)\right|^{2}}}
$$

and displayed for the last iteration step in Table 1

The agreement is obviously excellent and below $2 \%$ for all three cases. It is noteworthy that the error for the velocity is smaller than the one for the temperature. When increasing the discrete velocity directions for the temperature distribution functions from 6 to 9 , this error should decrease. Figure 4 plots the averaged error for the computed macroscopic velocity and temperature over the computational iteration steps. The convergence to a fixed value is obvious.

Table 1. Spatial averaged error: the porous plate problem.

\begin{tabular}{|lll|}
\hline $\operatorname{Re}$ & $E_{\text {ave }}(u)[\%]$ & $E_{\text {ave }}(T)[\%]$ \\
\hline \hline 5 & 1.08 & 1.14 \\
10 & 0.64 & 0.98 \\
20 & 0.19 & 0.38 \\
\hline
\end{tabular}

3.2. Fluid flow past a heated rotating cylinder. In order to test the dynamic adaptation capabilities and boundary conditions for embedded complex geometries, we study the setup of a two-dimensional fluid flow past a heated isothermal rotating cylinder. The origin of the coordinate system is located at the center of the cylinder. As shown in Fig. 5, the left boundary is an inlet with constant temperature $T_{C}$, zero vertical velocity and constant inflow velocity $U_{\infty}$. On the right hand side of the domain, an outlet is modeled by imposing zero horizontal gradient boundary conditions for velocity and temperature. Slip adiabatic wall boundary conditions are applied at the upper and lower boundary. The cylinder boundary is modeled as a no-slip wall, which is isothermally heated to the constant temperature $T_{H}$ and 

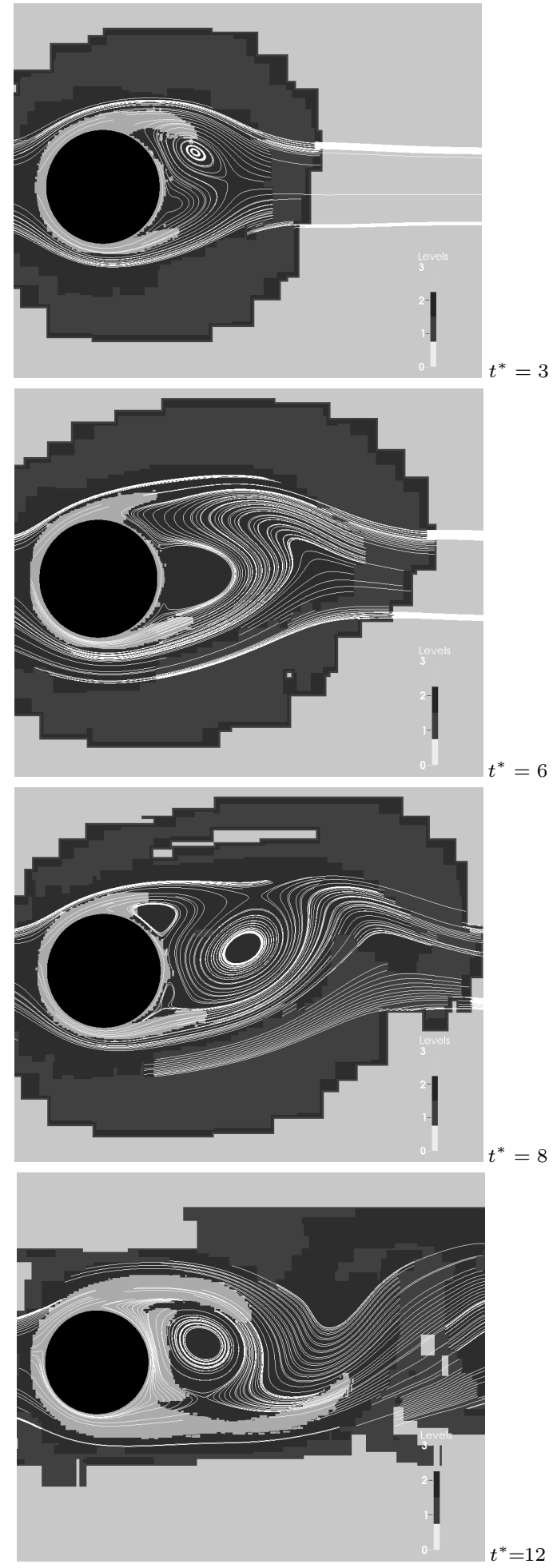

Fig. 6. Evolution of the velocity field and the adaptive mesh refinement regions for $\operatorname{Re}=200$ and $k=0.5$.

has the constant prescribed angular velocity $\Omega$. In terms of the cylinder radius $R=15$, the computational domain has the extensions $[-6 R, 16 R] \times[-8 R, 8 R]$, which is
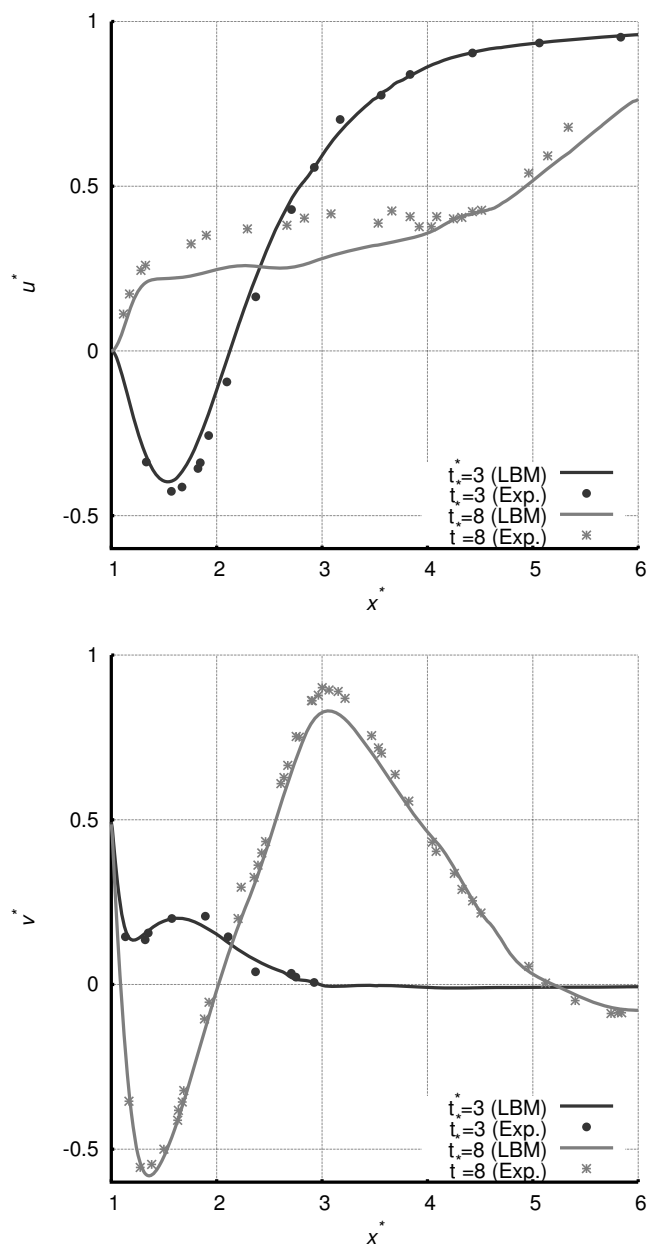

Fig. 7. Time evolution of the velocity components along the $x$-axis for $\operatorname{Re}=200$ and $k=0.5$.

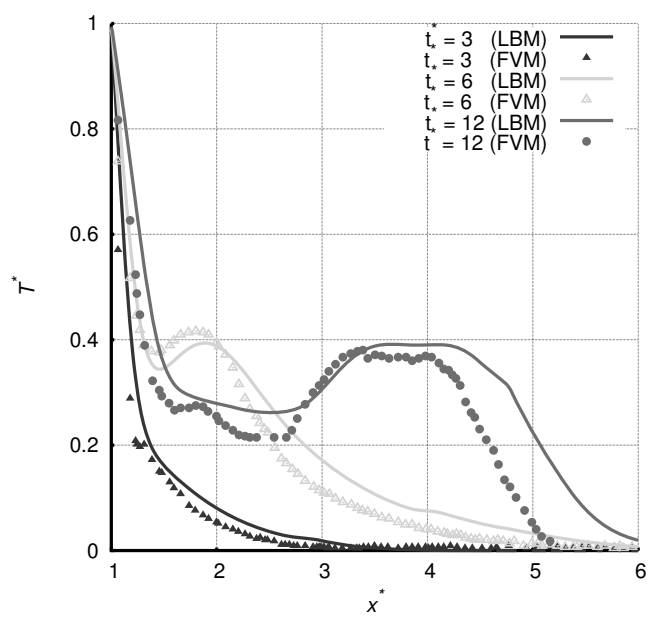

Fig. 8. Time evolution of the temperature along the $x$-axis for $\operatorname{Re}=200, \operatorname{Pr}=0.5$ and $k=0.5$.

sufficiently large to eliminate the boundary influences on the solution (Yan and $\mathrm{Zu}, 2008$ ). A base grid of $288 \times 240$ cells is used, and three additional levels refined by the factors $r_{1}=2$ for level 1 and $r_{2,3}=4$ for the other levels are applied. The dynamic refinement is based on 
scaled gradients of the velocity components as well as the temperature.

The entire velocity field is initialized as $\left(U_{\infty}, 0\right)^{T}$ and the temperature field is set as the constant value $T_{C}$. The Reynolds number is given by $\operatorname{Re}=2 U_{\infty} R / \nu$ and is set to $\operatorname{Re}=200$, where $U_{\infty}=0.01$ is used. The peripheral velocity $V$ of the rotating cylinder is given by $V=\Omega R$. With the parameter $k=V / U_{\infty}=0.5$ prescribed, we can determine $V$ and the angular velocity $\Omega$. To allow the direct comparison to the experimental results by Coutanceau and Menard (1985), the Prandtl number is set to $\operatorname{Pr}=0.5$ and all variables are normalized with the reference length $R$ and $U_{\infty}$ as velocity. Further, $\left(T-T_{C}\right) /\left(T_{H}-T_{C}\right)$ defines the reference temperature and the time normalization factor follows as $R / U_{\infty}$.

Figure 6 shows the dynamic adaptation during the computation at four different time points by displaying streamlines and the domains of different mesh refinement levels. The onset of vortex shedding can be inferred. The finest refinement level is located directly around the cylinder, namely, where the boundary layers are located and detach from the cylinders surface. The unrefined regions are in the outer regions of the domain. The refined levels move downstream with the shedding vortices and the cylinder wake increases over time.

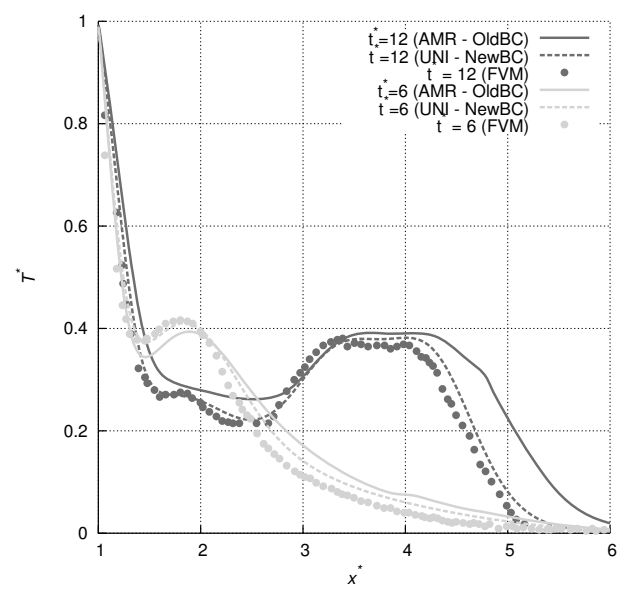

Fig. 9. Comparison of simulation results with different curved boundary conditions used: time evolution of the temperature along the $x$-axis for $\operatorname{Re}=200, \operatorname{Pr}=0.5$ and $k=0.5$.

Figure 7 compares the temporal evolution of the velocity components along representative points on the $x$-axis obtained in the simulation and with data from the experiment, while Fig. 8 displays the time evolution of the scalar temperature versus numerical results reported by Lai and Yan (2001). The latter adopted a finite volume method with non-orthogonal grids. Again, our simulation results are in good agreement with some differences in the $u$-velocity component at $t^{*}=8$ when the vortex is shed (see Fig. 6). A possible explanation is our rather simple temperature operator with only four discrete unity directions and with the employed boundary conditions for the curved boundary explained in Section 2.4 However, by using the bounce back scheme for curved moving boundaries of Bouzidi et al. (2001) and $\mathrm{Li}$ et al. (2013) with a global uniform mesh, the differences are considerably reduced (cf. Fig. 9). Therefore, the next step is to implement the curved boundary treatment in the AMR method.

3.3. Natural convection in a square 2D-cavity. In order to benchmark the overall method, we employ a two-dimensional square cavity with differentially heated walls. At the vertical walls, isothermal temperatures $T_{H}$ and $T_{C}$ are prescribed and adiabatic boundary conditions are applied at the top and the bottom. Further, at all four walls we prescribe no-slip boundary conditions for the velocity field. Figure 10 depicts this setup.

The flow is characterized by the Prandtl number $\operatorname{Pr}=0.71$ (air) and the Rayleigh numbers $\mathrm{Ra}=10^{j}$ with $j=3, \ldots, 8$ with accordingly increasing velocities $U$. The reference temperature is given by $T_{\text {ref }}=\left(T_{H}+\right.$ $\left.T_{C}\right) / 2$. The simulations were terminated after reaching steady state. Two additional levels of refinement with $r_{1,2}=2$ are used and the base mesh has $\left(H \Delta x_{0}\right)^{2}$ cells, whereby $\Delta x_{0}=1$ and $H$ is given in the left column of Table 2] For simulations with $\mathrm{Ra}=10^{3}, \ldots, 10^{6}$, we use the defined refinement thresholds for horizontal and vertical velocity $\epsilon_{u}, \epsilon_{v}$ with $2.5 \%$ of the characteristic velocity and $1 \%$ of the maximum temperature. The thresholds for $\mathrm{Ra}=10^{7}$ and $10^{8}$ remain as previously stated.

We compare our adaptive simulation results to published reference data by De Vahl Davis (1983), who solved the NS equations on a uniform square mesh with a second order finite difference method, and by Guo et al. (2002), who used the incompressible thermal LBGK approach presented above with a uniform mesh. Further results by Kuznik et al. (2007), who used a D2Q9 DDF LBM approach with non-uniform mesh resolution, are listed in Table 2 Table 2 contains the obtained maximal horizontal velocity $u_{\max }$ along the vertical center line at $x=H / 2$ and the location $y_{\max }$ of its occurrence and similarly for the horizontal center line at $y=H / 2$, the maximal vertical velocity $v_{\max }$ and its location $x_{\max }$. Furthermore, the average Nusselt number

$$
\mathrm{Nu}_{\text {ave }}=-\left.\int_{0}^{H} \frac{1}{\Delta T} \frac{\partial T}{\partial x}\right|_{x=0} \mathrm{~d} y
$$

is compared. Velocity values in Table 2 are normalized by the reference diffusion velocity $\mathcal{D} / H$. As expected, $u_{\max }$, $v_{\max }$ and $\mathrm{Nu}_{\text {ave }}$ increase with the increasing Rayleigh 
Table 2. Comparison of the simulation results: natural convection in the square cavity.

\begin{tabular}{|lcccccc|}
\hline & Ref. & $u_{\max }$ & $y_{\max }$ & $v_{\max }$ & $x_{\max }$ & $\mathrm{Nu}_{\text {ave }}$ \\
\hline \hline $\mathrm{Ra}=10^{3}$ & $\mathrm{a}$ & 3.640 & 0.810 & 3.688 & 0.180 & 1.115 \\
$U=0.01$ & $\mathrm{~b}$ & 3.649 & 0.813 & 3.697 & 0.178 & 1.114 \\
$H=100$ & $\mathrm{c}$ & 3.655 & 0.813 & 3.699 & 0.180 & 1.115 \\
& $\mathrm{~d}$ & 3.636 & 0.809 & 3.686 & 0.174 & 1.117 \\
\hline $\mathrm{Ra}=10^{4}$ & $\mathrm{a}$ & 16.161 & 0.823 & 19.595 & 0.118 & 2.239 \\
$U=0.02$ & $\mathrm{~b}$ & 16.178 & 0.823 & 19.617 & 0.119 & 2.245 \\
$H=150$ & $\mathrm{c}$ & 16.076 & 0.820 & 19.637 & 0.117 & 2.248 \\
& $\mathrm{~d}$ & 16.167 & 0.821 & 19.597 & 0.120 & 2.246 \\
\hline $\mathrm{Ra}=10^{5}$ & $\mathrm{a}$ & 34.666 & 0.855 & 68.457 & 0.066 & 4.504 \\
$U=0.05$ & $\mathrm{~b}$ & 34.730 & 0.855 & 68.590 & 0.066 & 4.510 \\
$H=200$ & $\mathrm{c}$ & 34.834 & 0.859 & 68.267 & 0.062 & 4.535 \\
& $\mathrm{~d}$ & 34.962 & 0.854 & 68.578 & 0.067 & 4.518 \\
\hline $\mathrm{Ra}=10^{6}$ & $\mathrm{a}$ & 64.756 & 0.850 & 220.125 & 0.038 & 8.804 \\
$U=0.05$ & $\mathrm{~b}$ & 64.630 & 0.850 & 219.360 & 0.038 & 8.806 \\
$H=200$ & $\mathrm{c}$ & 65.361 & 0.852 & 216.415 & 0.039 & 8.778 \\
& $\mathrm{~d}$ & 64.133 & 0.860 & 220.537 & 0.038 & 8.792 \\
\hline $\mathrm{Ra}=10^{7}$ & $\mathrm{a}$ & 140.255 & 0.887 & 702.459 & 0.021 & 16.429 \\
$U=0.05$ & $\mathrm{~d}$ & 148.768 & 0.881 & 702.029 & 0.020 & 16.408 \\
$H=256$ & & & & & & \\
\hline $\mathrm{Ra}=10^{8}$ & $\mathrm{a}$ & 297.145 & 0.945 & 2228.413 & 0.012 & 29.954 \\
$U=0.05$ & $\mathrm{~d}$ & 321.457 & 0.940 & 2243.36 & 0.012 & 29.819 \\
$H=256$ & & & & & & \\
\hline $\mathrm{a}=\mathrm{present}(\mathrm{LBM}$-AMROC), $\mathrm{b}=$ De Vahl Davis(1983) (FDM: uniform),
\end{tabular}

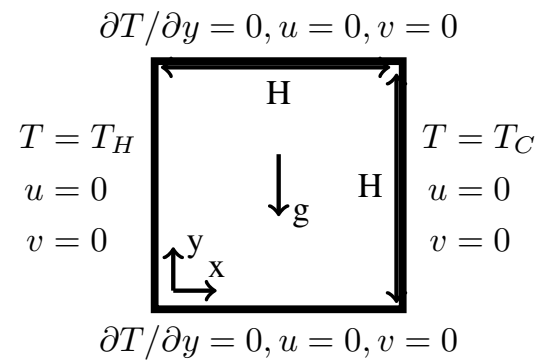

Fig. 10. Configuration of the two dimensional cavity.

number Ra. Comparing the $\mathrm{Nu}$ numbers predicted by our adaptive method to the literature data, an agreement within $2 \%$ is found for all Ra numbers. Figure 11 shows the vertical velocity component in the horizontal mid-plane for all discussed Rayleigh numbers. The velocity profiles plotted in Fig. 11 reveal the development of a boundary layer close to the heated/cooled walls with velocity maxima/minima whose values increase/decrease with increasing/decreasing $\mathrm{Ra}$. This increase in the magnitude of the vertical velocity with increasing $\mathrm{Ra}$ is also reflected in Table 2, To give an impression of the flow solution, contours of the temperature fields and streamlines are presented in Fig. 12 for the three Ra numbers considered.

For all the three Ra numbers, the streamlines reflect that fluid rises at the heated wall and descends at the

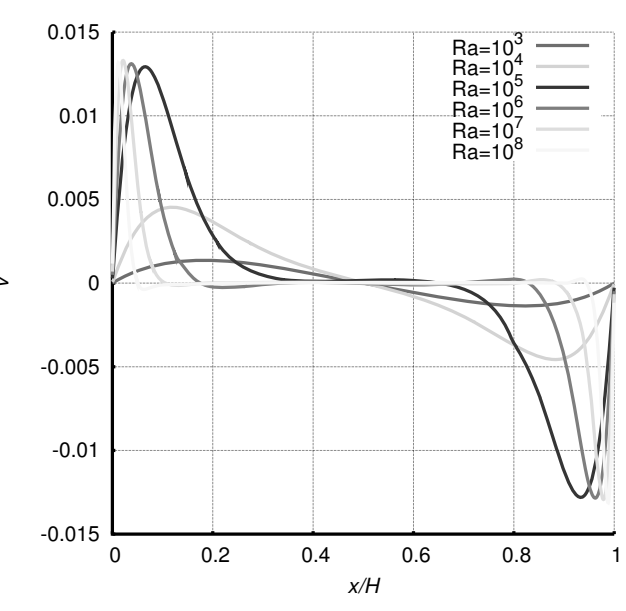

Fig. 11. Vertical velocity in the horizontal mid-plane of the 2D cavity for different Rayleigh numbers.

cooled wall. This generates a circulation around the center where the velocity is zero. For the lower Ra numbers, the computed flow field are in good agreement with results reported in previous studies (De Vahl Davis, 1983; Guo et al., 2002; Azwadi Che Sidik and Irwan, 2010; Kuznik et al., 2007; Abdelhadi et al., 2006). On the graph with the contours predicted for $\mathrm{Ra}=10^{7}$, the mesh refinement 


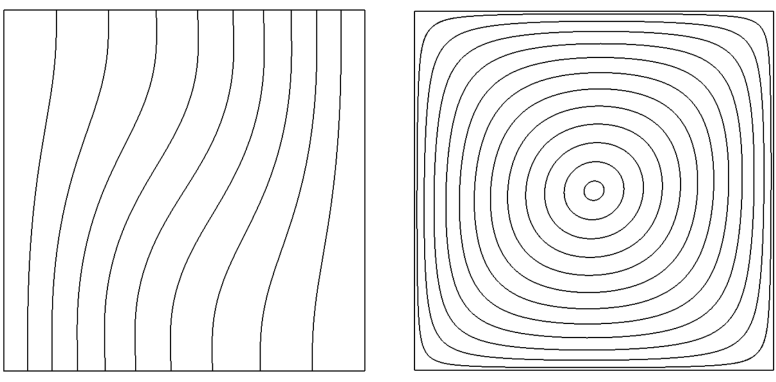

$\mathrm{Ra}=10^{3}$

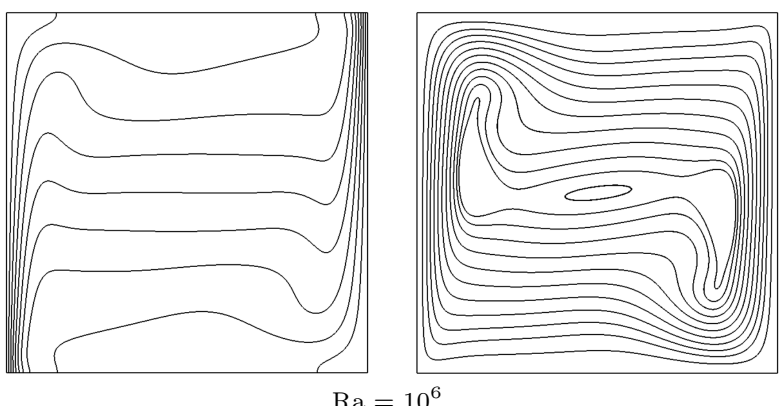

$\mathrm{Ra}=10^{6}$
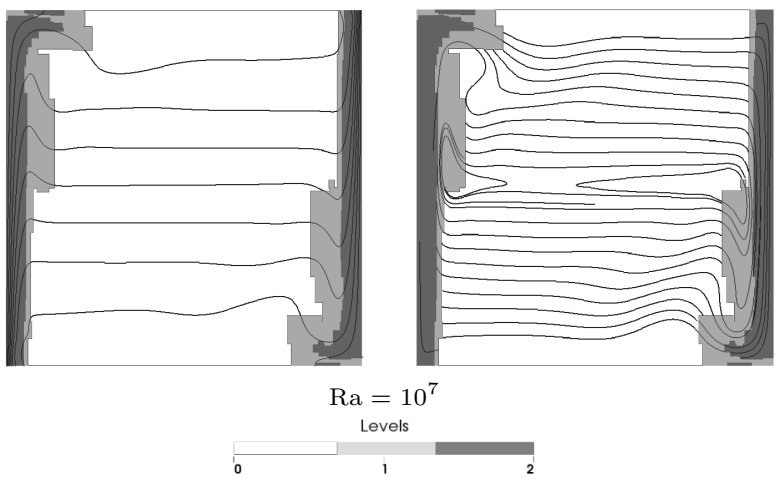

Fig. 12. LBM results of natural convective flow in the square cavity for three Ra numbers. Left: contours of isotherms, right: streamlines.

levels realized in the domain are additionally highlighted by grayscales. From the predominantly vertical isotherms obtained for the of a case low Ra number, it can be concluded that the heat conduction dominates the heat transport between the heated walls. For larger Ra the isotherms are aligned more horizontally in the cavity's center due to the thinner boundary layers. The denser isotherms near the hot and cold walls further reflect the lower thermal boundary layer thickness for a higher Rayleigh number. It is in this region that on-the-fly mesh resolution is particularly beneficial.

3.4. Natural convection in a cubic cavity. To benchmark the three-dimensional implementation of the method, we employ a 3D cubic cavity with differentially heated walls. As before, at the vertical walls, the constant temperatures $T_{H}$ and $T_{C}$ are prescribed. At the bottom, top and front, back walls adiabatic boundary conditions

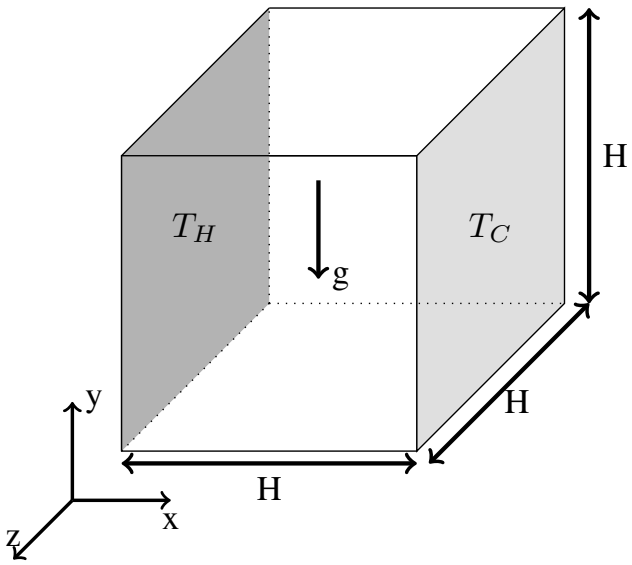

Fig. 13. Configuration of the three dimensional cavity.

are used for the temperature, while no-slip boundary conditions at all six walls are realized for the velocity fields. In summary, Fig. 13 represents this numerical setup.

Again, the Prandtl number is $\operatorname{Pr}=0.71$ (air) and in the $3 \mathrm{D}$ simulations the Rayleigh number $\mathrm{Ra}=10^{j}$ is varied from $j=3, \ldots, 5$. Here, we focus on the flow for $\mathrm{Ra} \leq 10^{5}$, since for higher $\mathrm{Ra}$ the flow is expected to become unsteady and eventually turbulent. To benchmark our method for a turbulent flow is, however, beyond the scope of this paper. As discussed above, the buoyancy (reference) velocity $U$ rises with increasing $\mathrm{Ra}$ and the reference temperature is given by $T_{\text {ref }}=$ $\left(T_{H}+T_{C}\right) / 2$. Two additional levels of refinement with $r_{1}=2, r_{2}=4$ are used and the base mesh has $\left(H \Delta x_{0}\right)^{3}$ cells, whereby $\Delta x_{0}=1$ and $H$ is given in the left column of Table 3 The adaptive mesh refinement obeys the scaled gradient criteria given above in (18). The thresholds used for all three velocity components are $1 \%, 2 \%$ and $5 \%$ of the reference velocity $U$ for $\mathrm{Ra}=10^{3}, 10^{4}$ and $10^{5}$, respectively. As before, $1 \%$ of $T_{H}$ is employed as the temperature refinement threshold. The computed results are compared with published literature results after reaching steady state.

Azwadi Che Sidik and Syahrullail (2009) use a D3Q19 DDF LBM approach with a D3Q6 operator for the temperature field and a uniform cubic mesh to get excellent numerical stability and accuracy. Peng et al. (2003) use a three-dimensional incompressible LBM with a DDF approach and two D3Q19 operators for the two fields and a non-uniform mesh resolution. Finally, Fusegi et al. (1991) use a high-resolution, finite difference NS solver with a uniform mesh resolution result and obtain results which agree reasonably well with experimental measurements. Figure 14 visualizes the temperature isosurfaces in the cubic enclosure and the different mesh refinement levels in the symmetry plane for $\mathrm{Ra}=$ $10^{4}, 10^{5}$. Near the heated walls, the isosurfaces are 
predominantly vertical. Notice that the isosurfaces in the center of the cavity become more horizontally with increasing $\mathrm{Ra}$. The reason is that the thermal boundary layer is becoming thinner. This observation is similar to that in the previous chapter. Note that the shaping of the mesh refinement levels for $\mathrm{Ra}=10^{4}$ is much more pronounced than for $\mathrm{Ra}=10^{5}$. As in the previous section, we compare the results on the symmetry plane $z=H / 2$ in terms of maximal horizontal velocity $u_{\max }$ along the vertical center line at $x=H / 2$ and at the corresponding location $y_{\max }$ of its occurrence, and similarly for the horizontal center line at $y=H / 2$, the maximal vertical velocity $v_{\max }$ and its location $x_{\max }$. Furthermore, we use the average Nusselt number (22) for comparison. Our results are listed in the table 3 . The velocity values in Table 3 are normalized with the reference velocity $U$.

The Nusselt number increases with the increasing Ra number, which means that the convective part of the heat transfer predominates the conduction. Comparing the $\mathrm{Nu}$ numbers predicted with our method to the literature, an agreement within $2 \%$ is found for all three Ra numbers, although the comparison of the horizontal velocity component shows larger differences. The reason for this might be a lack of dynamic mesh refinement near the upper and bottom walls. The mesh refinement is more pronounced near the heated and cooled walls, where the thinner thermal boundary layers are located.

\section{Conclusions}

A novel two and three dimensional incompressible dynamically adaptive thermal lattice Boltzmann method on block-based hierarchical finite volume meshes with embedded complex geometric structures has been developed and validated. The agreement for a two-dimensional porous plate problem on a Cartesian grid is nearly perfect. Successful validation against analytic solutions of the Navier-Stokes equations, e.g., for a heated rotating cylinder for $\mathrm{Pr}=0.5$ has been achieved. While for this particular example the deviations in velocity and temperature were found to increase over time, a possible improvement could be the implementation of a bounce-back boundary condition for curved boundaries.

For the benchmark of a two-dimensional heated cavity with Rayleigh numbers from $\mathrm{Ra}=10^{3}$ to $10^{8}$, the predictions are in good agreement with published results. Our results in the form of the computed Nusselt number reach an agreement within $2 \%$. For higher Rayleigh numbers, the deviations in the quantities considered are greater in regions without refinement. The comparison for a three-dimensional heated cubic cavity with Rayleigh numbers from $\mathrm{Ra}=10^{3}$ to $10^{5}$ against literature results delivers a good agreement as well. In terms of the Nusselt number, the agreement with literature results is again
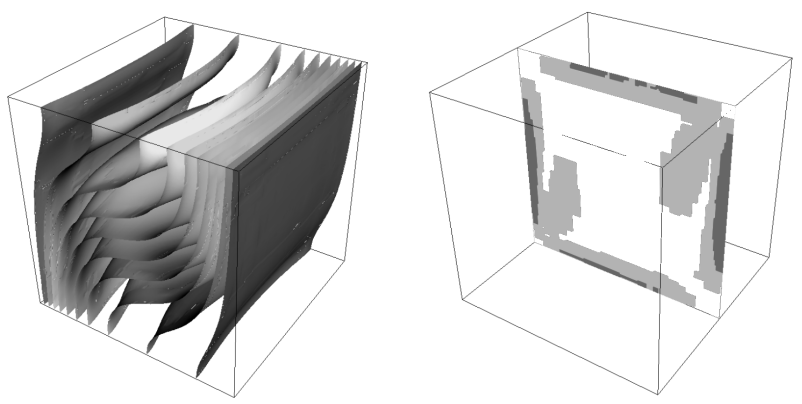

$\mathrm{Ra}=10^{4}$

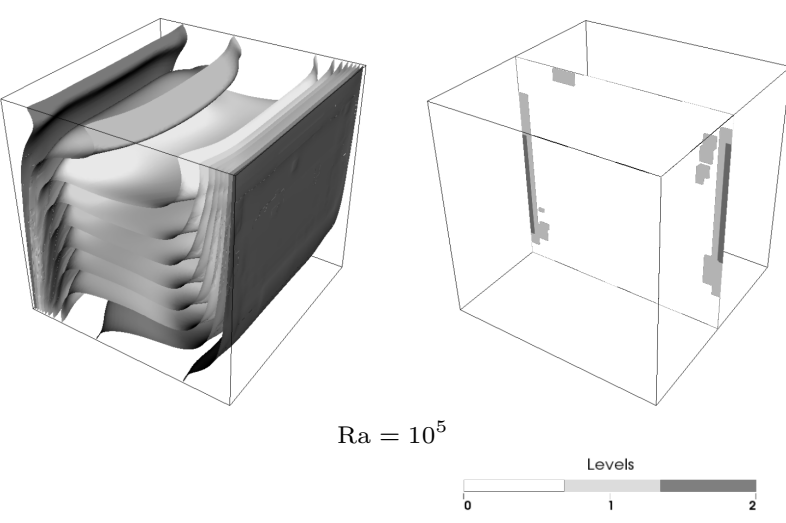

Fig. 14. Simulation results of natural convective flow in the cubic cavity. Left: isosurfaces of temperature (gray scale indicates temperature), right: mesh refinement levels.

under $2 \%$. A comprehensive analysis of CPU-time and memory savings by employing our unique block-based adaptive LBM will be conducted in the future. We will also take a closer look at how the results are influenced by the refinement criteria. Finally, extension and validation of the 3D approach to turbulent flows at higher Ra or Re numbers is planned.

\section{References}

Abdelhadi, B., Hamza, G., Razik, B. and Raouache, E. (2006). Natural convection and turbulent instability in cavity, WSEAS Transactions on Heat and Mass Transfer 1(2): 179-184.

Alexander, F.J., Chen, S. and Sterling, J.D. (1993). Lattice Boltzmann thermohydrodynamics, Physical Review E 47: 2249-2252.

Azwadi Che Sidik, N. and Irwan, M. (2010). Simplified mesoscale lattice Boltzmann numerical model for prediction of natural convection in a square enclosure filled with homogeneous porous media, WSEAS Transactions on Fluid Mechanics 3(5): 186-195.

Azwadi Che Sidik, N. and Syahrullail, S. (2009). A three-dimension double-population thermal lattice BGK model for simulation of natural convection heat transfer in a cubic cavity, WSEAS Transactions on Mathematics 8(9): 561-571. 
Table 3. Comparison of the simulation results: natural convection in the cubic cavity.

\begin{tabular}{|lcccccc|}
\hline & Ref. & $u_{\max }$ & $y_{\max }$ & $v_{\max }$ & $x_{\max }$ & $\mathrm{Nu}_{\text {ave }}$ \\
\hline \hline $\mathrm{Ra}=10^{3}$ & $\mathrm{a}$ & 0.132 & 0.195 & 0.132 & 0.829 & 1.099 \\
$U=0.01$ & $\mathrm{e}$ & 0.132 & 0.186 & 0.132 & 0.841 & 1.096 \\
$H=81$ & $\mathrm{f}$ & 0.132 & 0.188 & 0.133 & 0.826 & 1.097 \\
& $\mathrm{~g}$ & 0.131 & 0.200 & 0.132 & 0.833 & 1.105 \\
\hline $\mathrm{Ra}=10^{4}$ & $\mathrm{a}$ & 0.197 & 0.194 & 0.220 & 0.887 & 2.270 \\
$U=0.02$ & $\mathrm{e}$ & 0.200 & 0.182 & 0.224 & 0.883 & 2.301 \\
$H=81$ & $\mathrm{f}$ & 0.206 & 0.163 & 0.221 & 0.887 & 2.304 \\
& $\mathrm{~g}$ & 0.201 & 0.183 & 0.225 & 0.883 & 2.302 \\
\hline $\mathrm{Ra}=10^{5}$ & $\mathrm{a}$ & 0.141 & 0.152 & 0.242 & 0.935 & 4.583 \\
$U=0.1$ & $\mathrm{e}$ & 0.151 & 0.142 & 0.248 & 0.930 & 4.670 \\
$H=91$ & $\mathrm{f}$ & 0.149 & 0.136 & 0.240 & 0.935 & 4.658 \\
& $\mathrm{~g}$ & 0.147 & 0.145 & 0.247 & 0.935 & 4.646 \\
\hline
\end{tabular}

$\mathrm{a}=$ present (LBM-AMROC), e = Azwadi Che Sidik and Syahrullail (2009) (LBM: uniform),

f = Peng et al. (2003) (LBM: non-uniform), g= Fusegi et al. (1991) (NS: uniform).

Berger, M. and Colella, P. (1988). Local adaptive mesh refinement for shock hydrodynamics, Journal of Computational Physics 82(1): 64-84.

Bhatnagar, P., Gross, E. and Krook, M. (1954). A model for collisional processes in gases, I: Small amplitude processes in charged and in neutral one-component systems, Physical Review 94(3): 511-525.

Bouzidi, M., Firdaouss, M. and Lallemand, P. (2001). Momentum transfer of a Boltzmann-lattice fluid with boundaries, Physics of Fluids 13(11): 3452-3459.

Chen, H., Filippova, O., Hoch, J., Molvig, K., Shock, R., Teixeira, C. and Zhang, R. (2006). Grid refinement in lattice Boltzmann methods based on volumetric formulation, Physica A 362(1): 158-167.

Chen, H. and Teixeira, C. (2000). H-theorem and origins of instability in thermal lattice Boltzmann models, Computer Physics Communications 129(1): 21-31.

Chen, S. and Doolen, G. (1998). Lattice Boltzmann method for fluid flows, Annual Review of Fluid Mechanics 30: 329-364.

Coutanceau, M. and Menard, C. (1985). Influence of rotation on the near-wake development behind an impulsively started circular cylinder, Journal of Fluid Mechanics 158: 399-446.

De Vahl Davis, G. (1983). Natural convection of air in a square cavity a benchmark numerical solution, International Journal for Numerical Methods in Fluids 3(3): 249-264.

Deiterding, R. (2011). Block-structured adaptive mesh refinement-theory, implementation and application, ESAIM Proceedings 34: 97-150.

Deller, P. (2002). Lattice kinetic schemes for magnetohydrodynamics, Journal of Computational Physics 179(1): 95-126.

Dupuis, A. and Chopard, B. (2003). Theory and applications of an alternative lattice Boltzmann grid refinement algorithm, Physica E 67: 066707.
Fusegi, T., Hyun, J., Kuwahara, K. and Farouk, B. (1991). A numerical study of three-dimensional natural convection in a differentially heated cubical enclosure, International Journal of Heat and Mass Transfer 34(6): 1543-1557.

Guo, Z., Shi, B. and Zheng, C. (2002). A coupled lattice BGK model for the Boussinesq equations, International Journal for Numerical Methods in Fluids 39(4): 325-342.

Hähnel, D. (2004). Molekulare Gasdynamik, Springer, Heidelberg.

He, N.-Z., Wang, N.-C., Shi, B.-C. and Guo, Z.-L. (2004) A unified incompressible lattice BGK model and its application to three-dimensional lid-driven cavity flow, Chinese Physics 13(1): 40-46.

He, X., Chen, S. and Doolen, G. (1998). A novel thermal model for the lattice Boltzmann method in incompressible limit, Journal of Computational Physics 146(1): 282-300.

He, X. and Luo, L.-S. (1997). Lattice Boltzmann model for the incompressible Navier-Stokes equation, Journal of Statistical Physics 88(3): 927-944.

Jonas, L., Chopard, B., Succi, S. and Toschi, F. (2006). Numerical analysis of the average flow field in a turbulent lattice Boltzmann simulation, Physica A 32(1): 6-10.

Kuznik, F., Vareilles, J., Rusaouen, G. and Kraiss, G. (2007). A double-population lattice Boltzmann method with non-uniform mesh for the simulation of natural convection in a square cavity, International Journal of Heat and Fluid Flow 28(5): 862-870.

Lai, H. and Yan, Y. (2001). The effect of choosing dependent variables and cell-face velocities on convergence of the SIMPLE algorithm using non-orthohonal grids, International Journal of Numerical Methods for Heat \& Fluid Flow 11(6): 524-546.

Lee, T. and Lin, C. (2005). A stable discretization of the lattice Boltzmann equation for simulation of incompressible two-phase flows at high density ratio, Journal of Computational Physics 206(1): 16-47. 
Li, L., Mei, R. and Klausner, J. (2013). Boundary conditions for thermal lattice Boltzmann equation method, Journal of Computational Physics 237(1): 366-395.

McNamara, G. and Alder, B. (1993). Analysis of lattice Boltzmann treatment of hydrodynamics, Physica A 194(1): 218-228.

Mohamad, A. (2011). Lattice Boltzmann MethodFundamentals and Engineering Applications with Computer Codes, Springer, London.

Mohamad, A. and Kuzmin, A. (2010). A critical evaluation of force term in lattice Boltzmann method, natural convection problem, International Journal of Heat and Mass Transfer 53: 990-996.

Peng, Y., Shu, C. and Che, Y. (2003). A 3D incompressible thermal lattice Boltzman model and its application to simulation natural convection in a cubic cavity, Journal of Computational Physics 193(1): 260-274.

Qian, Y. (1993). Simulating thermohydrodynamics with lattice BGK models, Journal of Scientific Computing 8(3): 231-241.

Qian, Y., D'Humires, D. and Lallemand, P. (1992). Lattice BGK models for Navier-Stokes equation, Europhysics Letters 17(6): 479-484.

Succi, S. (2001). The Lattice Boltzmann Equation: For Fluid Dynamics and Beyond, Numerical Mathematics and Scientific Computation, Clarendon Press, Oxford.

Yan, Y. and Zu, Y. (2008). Numerical simulation of heat transfer and fluid flow past a rotating isothermal cylinder-A LBM approach, International Journal of Heat and Mass Transfer 51(9-10): 2519-2536.

Yu, Z. and Fan, L.-S. (2009). An interaction potential based lattice Boltzmann method with adaptive mesh refinement (AMR) for two-phase flow simulation, Journal of Computational Physics 230(17): 6456-6478.

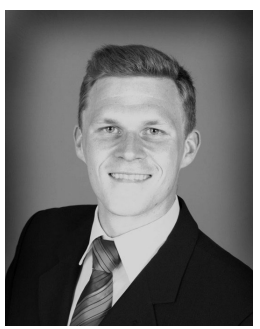

Kai Arne Feldhusen was born in Germany in 1986. He received his diploma degree in industrial mathematics from the University of Bremen in 2013. Afterwards, he worked at the Institute of Aerodynamics and Flow Technology at the German Aerospace Center in Göttingen on the development of a lattice Boltzmann solver for thermal convection problems in curved, threedimensional geometries. Currently, he is in a teacher training program for a secondary school

in Salzgitter, Germany.

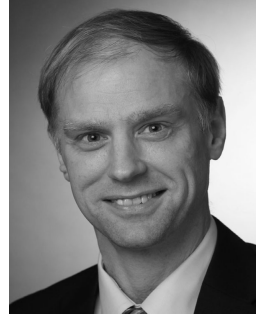

Ralf Deiterding was born in Germany in 1971. After high school and military service, he graduated with a diploma degree in industrial mathematics from the Technical University of Clausthal, Germany, in 1998. He obtained a Ph.D. in applied mathematics and computational fluid dynamics from Brandenburg Technical University in Cottbus, Germany, in 2003. Between 2003 and 2006 he was a postdoctoral scholar in applied and computational mathematics and aerospace engineering at the California Institute of Technology in Pasadena, USA. He joined the US Department of Energy in the Oak Ridge National Laboratory in 2006 as a householder fellow in scientific computing and worked in the laboratory until 2013 as a staff-level mathematician. Between 2013 and 2015 he was a group leader for computational fluid dynamics in the Department of Fluid Systems at the German Aerospace Center (DLR) in Göttingen, Germany. Since mid-2015 he has been an associate professor of fluid dynamics at the University of Southampton, UK.

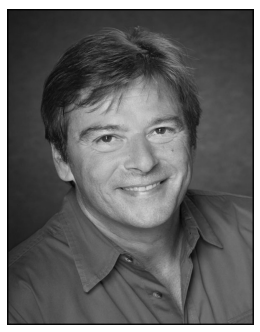

Claus Wagner was born in 1962 in Germany. In 1983 he graduated from high school. After military service he received his Bachelor's degree (Vordiplom) in aerospace technologies from the University of the Armed Forces in 1986 and his Master's degree (Diplom-Ingenieur) in mechanical engineering from the University of Technology in Munich, Germany, in 1989. From 1990 to 1991 he stayed at the University of Florida in Gainesville, USA, and the NASA Langley Research Center in Hampton, USA. Afterwards he started his Ph.D. studies, which he finished in 1995 at the University of Technology in Munich, where he worked for another three years as a postdoc, until he moved to the German Aerospace Center (DLR) in Göttingen in 1998. In 2004, he received his habilitation from the University of Technology in Munich and in 2005 was appointed an honorary professor by the University of Technology in Ilmenau, Germany. In 2007 he stayed at the Center of Turbulence Research in Stanford, USA. Then he became the head of the Department of Fluid Systems at DLR in Göttingen, and in 2013 he was appointed a full professor and the head of the Aerodynamics Section at the University of Technology in Ilmenau, Germany.

Received: 30 September 2015 Revised: 3 May 2016 Accepted: 8 June 2016 